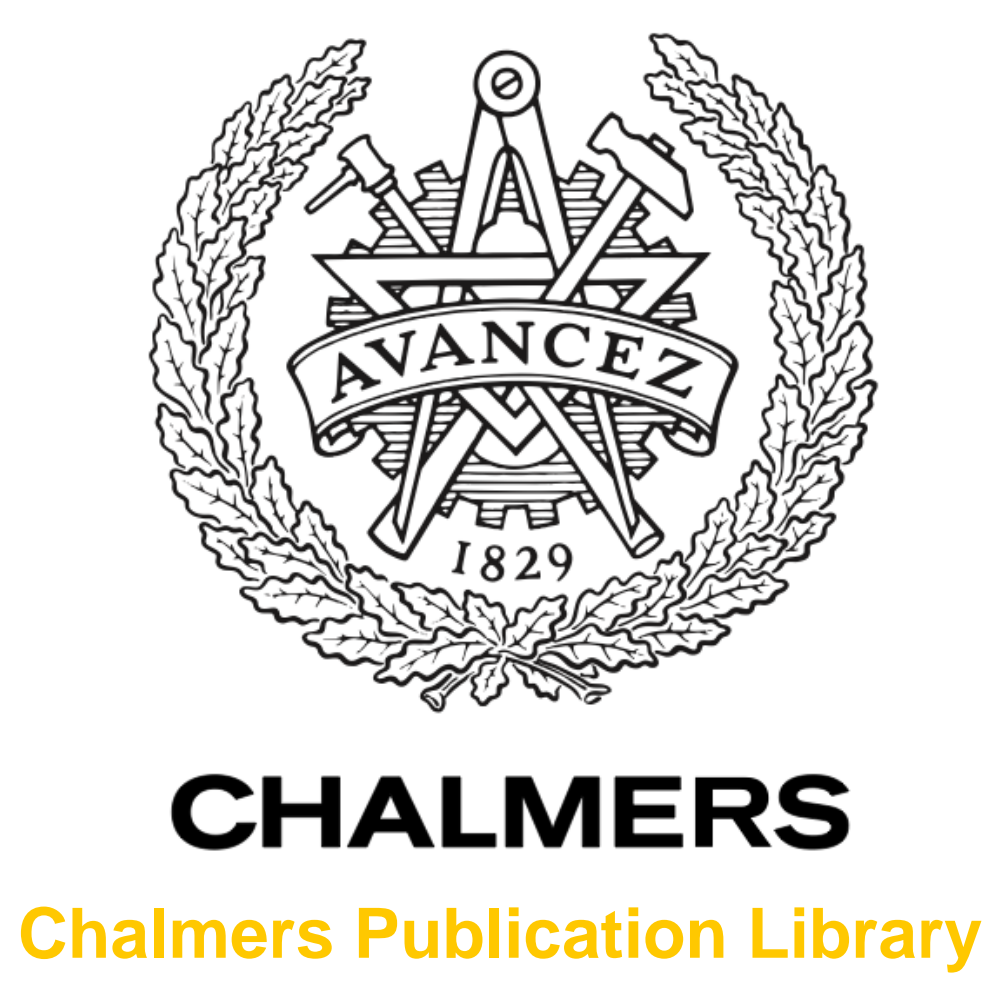

\title{
The effect of corrugation on heat transfer and pressure drop in channel flow with different Prandtl numbers
}

This document has been downloaded from Chalmers Publication Library $(\mathrm{CPL})$. It is the author's version of a work that was accepted for publication in:

International Journal of Heat and Mass Transfer (ISSN: 0017-9310)

Citation for the published paper:

Mirzaei, M. ; Davidson, L. ; Sohankar Esfahani, A. (2013) "The effect of corrugation on heat transfer and pressure drop in channel flow with different Prandtl numbers". International Journal of Heat and Mass Transfer, vol. 66 pp. 164-176.

Downloaded from: http://publications.lib.chalmers.se/publication/182783

Notice: Changes introduced as a result of publishing processes such as copy-editing and formatting may not be reflected in this document. For a definitive version of this work, please refer to the published source. Please note that access to the published version might require a subscription. 


\title{
The effect of corrugation on heat transfer and
}

\author{
pressure drop in channel flow with different
}

\section{Prandtl numbers}

Maryam Mirzaei*, Lars Davidson ${ }^{\dagger}$, Ahmad Sohankar $^{\ddagger}$, Fredrik Innings**

*Department of Mechanical Engineering, Yazd University, Yazd, Iran, m.mirzaei@stu.yazduni.ac.ir

${ }^{\dagger}$ Chalmers University of Technology, Gothenberg, Sweden, lada@chalmers.se

${ }^{\ddagger}$ Isfahan University of Technology, Isfahan, Iran, asohankar@cc.iut.ac.ir

${ }^{* *}$ CFD Manager at TetraPak, Lund, Sweden, fredrik.Innings@tetrapak.com

\begin{abstract}
Large Eddy Simulation and Direct Numerical Simulation are applied to study the turbulent flow field in a wavy channel at two Prandtl numbers, $\operatorname{Pr}=0.71$ and $\operatorname{Pr}=3.5$, and Reynolds number $R e_{b}=10000$. The characteristics of the separated shear layer and the near wall recirculating zone are discussed in relation to the turbulent heat transfer. Special attention is paid to the behavior of the flow and thermal boundary layers and various turbulent characteristics and their effects on the distribution of the Nusselt number and friction coefficient in the separation and reat-
\end{abstract}


tachment regions. The results indicate that the thickness of the thermal boundary layer rather than the turbulent fluctuations has a significant effect on the local variation of the averaged Nusselt number. The results are compared with Direct Numerical Simulation results of a plane channel at the same Reynolds number.

Keywords: Wavy channel, Plane channel, Turbulent heat transfer, Boundary layer, LES

\section{Introduction}

In various heat transfer devices, optimization of wall heat transfer is a key requirement to make the devices more compact or effective. The use of the surface protuberances is a passive heat transfer augmentation method and is based on developing boundary layers or streamwise fluctuations, creating swirl or vortices and flow destabilization or turbulence intensification . One typical technique is to use corrugations (waves) on the wall. Providing considerable heat transfer enhancement with a relatively low pressure drop as well as the simplicity of the manufacture method make these geometries more attractive than other passive enhancement methods such as ribs or vortex generators. The periodic changes of pressure gradient and the streamline curvature effects produce turbulence structures. The corrugated wall enhances heat transfer by destroying or decreasing the thermal boundary layer thickness and increasing the bulk flow mixing. The recirculating bubble in the wavy part of the channel and the separated shear layer formed above the separation point raise the turbulence intensity near the wall. The corrugated channels can be considered to be fully corrugated (fully- 
wavy or sinusoidal) or half corrugated channels. In a fully corrugated channel, the corrugation is applied to the entire channel length whereas in a half corrugated channel, part of the channel is corrugated and part of it remains smooth (see figure 1). The half corrugated channel is chosen as the geometry in the present study (referred to as the wavy channel).

Various studies have been conducted on turbulent flow over the fully corrugated walls [1]-[5]. Hudson et al. (1996) [1], Cherukat et al. (1998) [2] and Choi et al. (2005) [3] studied the turbulent flow in a fully wavy channel with experimental, Direct Numerical Simulation $(D N S)$ and Large Eddy Simulation (LES) methods, respectively. They provided an extensive study of different turbulent flow characteristics such as Reynolds shear stress, turbulent intensities and turbulence production over a wave with a wave amplitude to wave length ratio of 0.05. Calhoun et al. (2001) [4], Dellil et al. (2004) [5] and Yoon et al. (2009) [6] carried out numerical investigations of the effect of the wave amplitude in a fully wavy channel. They used different wave amplitude to wave length ratios and compared the turbulent flow patterns, the location of the separation and reattachment points and the mean distributions of heat transfer and pressure drop along the channel. As it has been mentioned in the literature review, most of the previous studies have been concentrated on the flow in a fully wavy channels, whereas in the present study a half corrugated channel is considered as the computational domain (see figure 1). Comparing the results of both geometries, some similarities and differences can be identified. For the wavy channels with identical wave amplitudes (fully and half corrugated), a small bubble appears at the bottom of the wave, where its location is nearly the same 
for both cases. In both cases, the peak values of the averaged Nusselt number and friction coefficient distributions occur around the separation and reattachment points. However, in half corrugated channel (the present study), due to the presence of the smooth part of the channel after the wave, the boundary layer formed after the reattachment point continues downstream of the wave, while for the fully wavy channel the boundary layer continues only until the next wave. The available studies concentrated mainly on the investigation of turbulent flow field whereas the present study pays detailed attention to both turbulent flow and temperature fields. We aim here to provide insight into the characteristics of the flow and heat transfer in a wavy channel by using Large eddy simulation $(L E S)$ and Direct Numerical Simulation $(D N S)$. This study particularly discusses the variation of the temperature and velocity boundary layers for two different Prandtl numbers and their effects on the heat transfer. The results are also compared with the plane channel flow.

The paper is organized as follows. A presentation of the numerical method, boundary conditions, the turbulence modelling, the computational domain and the grid is given in section 2 . Section 3 is devoted to a description of the results including an analysis of the global, time-averaged and instantaneous results for both plane and wavy channels. Some conclusions are drawn in the final section. 


\section{The modeling methodology}

\subsection{Governing equations}

The Navier-Stokes and energy equations for an incompressible viscous flow read:

$$
\begin{array}{r}
\frac{\partial \bar{u}_{i}}{\partial t}+\frac{\partial\left(\bar{u}_{i} \bar{u}_{j}\right)}{\partial x_{j}}=\beta \delta_{1 i}-\frac{1}{\rho} \frac{\partial \bar{P}}{\partial x_{i}}+\frac{\partial}{\partial x_{j}}\left[\left(\nu+\nu_{t}\right) \frac{\partial \bar{u}_{i}}{\partial x_{j}}\right] \\
\frac{\partial \bar{T}}{\partial t}+\frac{\left(\partial \bar{u}_{j} \bar{T}\right)}{\partial x_{j}}=\frac{\partial}{\partial x_{j}}\left[\left(\frac{\nu}{P r}+\frac{\nu_{t}}{P r_{t}}\right) \frac{\partial \bar{T}}{\partial x_{j}}\right]-\gamma \bar{u}_{1}
\end{array}
$$

$x_{1}, x_{2}, x_{3}(x, y, z)$ denote the Cartesian coordinate system, where the $x_{1}$ axis is aligned with the flow direction, the $x_{2}$ axis is the wall-normal direction and the $x_{3}$ axis is the spanwise direction. $\delta_{1 i}$ is the streamwise component of Kronecker delta.

The periodic boundary condition is employed in the streamwise and spanwise directions for both the plane and the wavy channel. Using the periodic boundary condition for the velocity and temperature requires special modifications in the Navier-Stokes equation in the $x$ direction and in the energy equation. The first term on the right-hand side of equation 1 represents the streamwise driving pressure gradient. For the plane channel, the Reynolds number based on the wall friction velocity $\left(R e_{\tau}\right)$ is prescribed, so $\beta$ is equal to 1 . For the wavy channel (see figure 1), to achieve the desired Reynolds number based on the bulk velocity $\left(R e_{b}\right), \beta$ is defined on the basis of the flow rate and is modified in each time step. The last term on the right-hand side of the energy equation provides the temperature gradient in the streamwise direction. For the constant heat flux $\left(q_{w}\right)$ condition on the wall, $\gamma$ is obtained based on the inlet and outlet 
energy balance as $\gamma=\frac{q_{w}}{\rho u_{r e f} H c_{p}}$ where $u_{r e f}$ is the wall friction velocity $\left(u_{\tau}\right)$ for the plane channel and the bulk velocity $\left(u_{b}\right)$ for the wavy channel.

For the wavy channel, the symmetry boundary condition is used for the upper wall and the no-slip condition and uniform heat flux are employed for the lower wall. For the plane channel, the no-slip condition and uniform heat flux are applied at both the upper and lower walls.

\subsection{Model description}

The present study uses the WALE turbulence model [8] as the subgrid scale model which reads:

$$
\begin{array}{r}
\nu_{s g s}=\left(C_{s} \Delta\right)^{2} \frac{\left(s_{i j}^{d} s_{i j}^{d}\right)^{3 / 2}}{\left(\bar{s}_{i j} \bar{s}_{i j}\right)^{5 / 2}+\left(s_{i j}^{d} s_{i j}^{d}\right)^{5 / 4}} \\
s_{i j}^{d}=\frac{1}{2}\left(\bar{g}_{i j}^{2}+\bar{g}_{j i}^{2}\right)-\frac{1}{3} \delta_{i j} \bar{g}_{k k}^{2}, \quad \bar{s}_{i j}=\frac{1}{2}\left(\frac{\partial \bar{u}_{i}}{\partial x_{j}}+\frac{\partial \bar{u}_{j}}{\partial x_{i}}\right)
\end{array}
$$

where $C_{s}=0.325, \bar{g}_{i j}$ is the velocity gradient tensor and $s_{i j}^{d}$ is the traceless symmetric part of the square of velocity gradient tensor.

\section{$2.3 \quad$ Numerical method}

An incompressible finite volume code based on an implicit, fractional step technique with a multigrid pressure Poisson solver and a non-staggered grid arrangement is employed [9]. The second order Crank-Nicolson scheme is used for time discretization. The spatial discretization is based on the second-order central differencing scheme. 


\subsection{Computational domain and grid influence}

The original domain is introduced as a periodic corrugated channel. To reduce computational costs, only one pitch of the domain is regarded as the computational domain. A two-dimensional schematic view (in the $x-y$ plane) of the three-dimensional computational domain and the corresponding geometrical parameters are displayed in figure 1 and table 1 , respectively. The shape of the lower wall in the wavy part is given by $y=-a \exp \left(-\left(\left(7 x-x_{0}\right)^{2}\right) /\left(2 \delta^{2}\right)\right)$, where $a=0.14, x_{0}=5.907$ and $\delta=1.8219$. The Reynolds number based on the bulk flow velocity and channel height is $R_{b}=u_{b} H / \nu=10000$. All the results presented below are normalized with bulk flow velocity, channel height, kinematic viscosity and bulk temperature $\left(T_{b}=q_{w} /\left(u_{b} \rho c_{p}\right)\right)$. Simulations are performed for two Prandtl numbers, $\operatorname{Pr}=0.71$ and 3.5. The time step is chosen as $\Delta t=0.005$. It should be mentioned that three different time steps, $\Delta t=0.005, \Delta t=0.01$ and $\Delta t=0.015$, have been tested in the present study and it was observed that the accuracy of the results becomes weaker, when a larger value of $\Delta t$ is employed. Therefore, $\Delta t=0.005$ is finally chosen as the proper time step. Based on the selected time step, the maximum local $C F L$ is less than 0.85 for all the simulations performed.

To find the proper grid, computations were made for four different grids. An overview of the grid resolutions is given in table 2. For all the grids presented, the $y^{+}$for the near wall nodes is less than 1 . Figure 2 displays a two-dimensional schematic view in the $x-y$ plane for the generated grid in the computational domain. The grid is displayed for every 4th cell. The global averaged Nusselt number, $\langle N u\rangle$, and friction coefficient, $\left\langle C_{f}\right\rangle$, for the different grids are compared 
in table 2 and, as can be seen, almost identical results are obtained for all the grids. The difference of $\left\langle C_{f}\right\rangle$ and $\langle N u\rangle$ are $2.8 \%$ between grids 1 and 3 and $4.8 \%$ and $1.7 \%$ between grids 1 and 4 , respectively.

Figure 3 compares the local variation of $\langle N u\rangle$ and $\left\langle C_{f}\right\rangle$ along the lower wall for four grids at $\operatorname{Pr}=0.71$. These results also reveal that the effect of using different grids is small. In grid 4 , the grid resolution is the same as in grid 2 but considering two pitches as the computational domain and, as seen, the results are very close. Hence, one pitch is chosen for the computational domain. Grid 3 is selected as the computational grid at $\operatorname{Pr}=0.71$. Figure 4 compares the streamwise variation of $\langle N u\rangle$ along the lower wall for two grids, grid 3 and grid 5 , at $\operatorname{Pr}=3.5$. In grids 3 and 5 , the number of nodes in the $x, y$ and $z$ directions is identical. Two different stretching factors in the $y$ direction are considered for grids 3 and 5 , where in grid 5 , the number of nodes from the wall to $y-y_{\text {wall }}=0.005$ is four times more than in grid 3 . The sizes of the cells near the upper boundary of the channel (the coarsest cells) are $\Delta y=0.0225$ and $\Delta y=0.025$ for grids 3 and 5 , respectively. Good agreement is observed between the grids 3 and 5 with less than $3 \%$ differences in major parts with a maximum difference of $15 \%$ occurring at the location of peak $N u$ number. Grid 5 is selected as the computational grid for $\operatorname{Pr}=3.5$.

Figure 5 compares the streamwise distributions of $\left\langle C_{f}\right\rangle$ and $\langle N u\rangle$ for the $L E S$ solution with the DNS results. In figure 5 (a), $\left\langle C_{f}\right\rangle$ obtained by $L E S$ for two grid resolutions $170 \times 198 \times 64$ (grid 3 in table 2) and $170 \times 82 \times 64$, referred as $L E S 1$ and $L E S 2$, are compared with the $D N S$ results of the same grid resolution as grid 3. As can be seen, by increasing the number of nodes in 
the $y$ direction the $L E S$ results become closer to the $D N S$ one. For the same grid resolution (grid 3), the LES predictions are in a good agreement with the $D N S$, which implies that the accuracy of the $L E S$ calculations is good.

As mentioned above, the flow is assumed to be periodic in both the $x$ and $z$ directions. Thus, the extent of the domain in the streamwise and spanwise directions should be chosen to be sufficiently large so that the spatial correlation of the velocity fluctuations drop to values close to zero. This indicates that the largest scales of turbulent structures are not affected by the finite domain size. Figure 6 presents the spanwise two point correlation, which is defined as $B_{w w}(\hat{z})=\left\langle w^{\prime}(z) w^{\prime}(z-\hat{z})\right\rangle /\left\langle w_{r m s}^{2}\right\rangle$. This figure compares the normalized two point correlation at three wall normal locations for two channel widths, $z_{\max }=1$ (using twice as many cells in the spanwise direction) and $z_{\max }=0.5$ (only half of the channel is plotted). It can be observed that for the channel with $z_{\max }=1$, the velocity correlations are very small before $z=0.25$ and drop to values below 0.1 . Figure 7 provides the streamwise distributions of $\left\langle C_{f}\right\rangle$ and $\langle N u\rangle$ for two channel width $z_{\max }=1$ and $z_{\max }=0.5$. Comparing the results for two different channel widths, a close agreement can be identified. For both $\left\langle C_{f}\right\rangle$ and $\langle N u\rangle$ the difference of the results are less than $4-5 \%$. Finally, $z_{\max }=0.5$ is chosen as the extent of the computational domain in the $z$ direction. 


\section{Results and Discussion}

\subsection{Plane channel}

To verify the accuracy of the computational method, a $D N S$ solution of the turbulent flow and heat transfer is performed for a plane channel flow. The size of the domain is chosen as $x_{\max }=3.2, y_{\max }=2$ and $z_{\max }=1.6$. The grid is geometrically stretched in the $y$ direction with a stretching factor of 1.15 from the wall to the symmetry line. The near-wall nodes are located at $y^{+} \approx 1$. The flow variables are normalized by the channel half height $(H)$, the friction velocity $\left(u_{\tau}\right)$, the kinematic viscosity $(\nu)$ and the friction temperature $\left(T_{\tau}=q_{w} /\left(u_{\tau} \rho c_{p}\right)\right)$. The Prandtl number is chosen as 0.71 and the Reynolds number based on the friction velocity and channel half height is $R e_{\tau}=395$.

The present $D N S$ results are compared with the $D N S$ results of Kawamura et al. [7] for plane channel flow at $R e_{\tau}=395$. The grid resolution and the mean flow variables obtained, such as the bulk velocity $\left(u_{b}\right)$, the bulk Reynolds number $\left(R e_{b}\right)$, the Nusselt number $(\langle N u\rangle)$ and friction coefficient $\left(\left\langle C_{f}\right\rangle\right)$ for both studies, are summarized in table 3 .

Figure 8 presents the mean velocity distribution $(8(\mathrm{a}))$, the root mean squared velocity fluctuations $(8(\mathrm{~b}))$ and the wall normal turbulent heat flux (8 (c)). Comparison of the results with the DNS results of Kawamura et al. [7] shows good agreement. 


\subsection{Wavy channel}

\subsubsection{Global characterizations}

Figure 9 presents the time and spanwise averaged streamlines in the wavy part of the channel, which displays a recirculation region formed at the bottom of the corrugation. Near the upper boundary of the channel, the streamlines are almost parallel and similar to those for the plane channel flow. As can be identified from the streamwise distribution of the averaged pressure in figure 10 (a), the presence of an adverse pressure gradient in the first half of the corrugation decreases the flow acceleration in the boundary layer near the wall, which leads to flow separation at $x=0.4$. The decreasing pressure accelerates the flow in the second half of the corrugation, and the flow reattaches to the wall at $x=1.1$. It should be noted that the pressure in Figure 10 (a) is periodic. The physical pressure, $p_{\text {phys }}$, is obtained as $p_{\text {phys }}=P-\beta x$, see equation 1 . Figure $10(\mathrm{~b})$ presents the root mean square of the pressure fluctuations $\left(P_{r m s}\right)$ at the wall in the wavy part of the channel. The maximum value is found near the reattachment point, and it corresponds to the location of the maximum of the mean pressure distribution (figure 10 (a)). The high pressure fluctuations around the reattachment point are probably a result of the impingement-like behavior at the reattachment point.

The streamwise distributions of the local Nusselt number, $(\langle N u\rangle)$, and friction coefficient, $\left(\left\langle C_{f}\right\rangle\right)$, for $\operatorname{Pr}=0.71$ and 3.5 are presented in figures 11 (a) and (b). Figure 11 (a) compares the maximum and minimum locations of the $\langle N u\rangle$ and $\left\langle C_{f}\right\rangle$ along the channel wall. All the values are scaled by the magnitudes 
at $x=0\left(\left\langle N u_{0}\right\rangle\right.$ and $\left.\left\langle C_{f 0}\right\rangle\right)$. The presence of the accelerated flow after the reattachment point and the flow impingement to the wall increase both $\langle N u\rangle$ and $\left\langle C_{f}\right\rangle$ after the reattachment point. As can be observed from figure 11 (a), the maximum values of $\langle N u\rangle$ and $\left\langle C_{f}\right\rangle$ are located at nearly the same locations (downstream of the reattachment point, at $x=1.282$ and at $x=1.325$, respectively). The minimum value of $\langle N u\rangle$ occurs near the separation point, $x=0.4$. In the smooth part of the channel, $x>1.7$, both the $\langle N u\rangle$ and $\left\langle C_{f}\right\rangle$ distributions become almost constant and slightly larger than those for the plane channel (7\% and $13 \%$ percent for $\operatorname{Pr}=0.71$ and 3.5 , respectively).

Figure 11 (b) compares the distribution of $\langle N u\rangle$ for $\operatorname{Pr}=0.71$ and $\operatorname{Pr}=3.5$. The results of the plane channel for both Prandtl numbers are also included, the $D N S$ results are presented for $\operatorname{Pr}=0.71$ and the analytical results of the Colburn equation $\left(\langle N u\rangle=0.023 \operatorname{Re}_{D}^{4 / 5} \operatorname{Pr}^{1 / 3}\right)$ are presented for both $\operatorname{Pr}=0.71$ and 3.5. This figure indicates that the distribution of $\langle N u\rangle$ and also the location of the maximum and minimum points for both Prandtl numbers are almost the same (the minimum occurs at $x=0.45$ for $\operatorname{Pr}=3.5$; note that the size of computational cell is $\Delta x=0.022)$. However, due to a thinner thermal boundary layer near the wall at $\operatorname{Pr}=3.5$ (see figure 21), the averaged Nusselt number is higher in this case than for $\operatorname{Pr}=0.71$. The local comparison of $\langle N u\rangle$ in both Prandtl numbers shows that the presence of corrugation in the channel causes different effects near the separation and reattachment points. For instance, the reduced magnitude between $x=0$ and the separation point $(x=0.4)$ is almost the same and about $40 \%$ while, between the reattachment point $(x=1.1)$ and the end of the pitch $(x=L)$, the increase is $21 \%$ and $36 \%$ for $\operatorname{Pr}=0.71$ and 
3.5 , respectively.

Beside the variations of the velocity and temperature boundary layers, different turbulent characteristics also affect the heat transfer (or $\langle N u\rangle)$ and pressure drop $\left(\right.$ or $\left.\left\langle C_{f}\right\rangle\right)$. Now the questions arise which of these parameters are more effective and whether these effects are the same for the two Prandtl numbers. To respond to these questions, a detailed investigation of the flow and thermal patterns in different parts of the channel will be described in the next sections.

\subsubsection{Flow field characterizations}

Figure 12 presents the normal distributions of the averaged velocity before the separation point, in the recirculation zone and after the reattachment point in the near wall region (figures $12(\mathrm{~b})-(\mathrm{d})$ ). The cross sections are displayed in figure 1 . The velocity distributions before the separation point, presented for the entire height of the channel, are also included in figure 12 (a). The results are compared with the $D N S$ results of the present study for the plane channel flow. As is seen, before the separation point, $0<x<0.3$, the velocity profiles are very similar. The presence of the recirculation zone near the wall for $0.4<x<1$, gives negative velocities very close to the wall. For $x>1.1$, the flow entirely moves forward again and the velocity is positive in the entire cross section. Considering the velocity profiles before the separation point (figure 12 (b)) and after the reattachment point (figure $12(\mathrm{~d})$ ), two distinct regions can be recognized near the wall for the velocity profiles in each cross section, i.e. a high wall-normal velocity gradient very close to the wall $\left(y-y_{\text {wall }} \lesssim 0.02\right)$, described as the inner region, and a low wall-normal velocity gradient (in comparison with 
the inner region), away from the wall that extend to the upper boundary of the channel, described as the outer region.

Before the separation point, $0<x<0.3$, the velocity gradient near the wall and the thickness of the inner regions are nearly independent of the streamwise direction; hence the boundary layer thickness is almost constant in this region. After the reattachment point, the velocity distributions are similar to those for the plane channel but with a different slope near the wall. For $x=1.1$, $x=1.2, x=1.4$ and $x=1.8$, the inner regions extend up to $y-y_{\text {wall }} \simeq 0.01$, $y-y_{\text {wall }} \simeq 0.02, y-y_{\text {wall }} \simeq 0.03$ and $y-y_{\text {wall }} \simeq 0.05$, respectively, which implies that a new boundary layer is formed with increasing thickness downstream of the reattachment point. It should be noted that the definition of an inner and outer region of the boundary layer is relevant only to the cross sections before the separation and after the reattachment points, (figure 12 (b)) and (figure 12 (d)), and does not include the sections inside the recirculation zone (figure 12 (c)).

Figures 13 (a), (b) and (c) present the contours of wall-normal velocity fluctuation $\left(\left\langle v^{\prime 2}\right\rangle\right)$, the Reynolds shear stress $\left(\left\langle u^{\prime} v^{\prime}\right\rangle\right)$ and the production of Reynolds shear stress $\left(\left\langle P_{12}\right\rangle\right)$, respectively, in the wavy part of the channel. From figure 13, a large region with high negative local values in $\left\langle u^{\prime} v^{\prime}\right\rangle$ and $\left\langle P_{12}\right\rangle$ is observed in the separated shear layer. This region is located above the recirculation zone; it begins close to the wall near $x=0.4$ and develops downstream until almost the end of the domain. Considering the $\left\langle v^{\prime 2}\right\rangle$ and $\left\langle u^{\prime} v^{\prime}\right\rangle$ contours, one can see that the minimum absolute values appear near the separation zone while the maximum absolute values occur around the reattachment zone. 
To further clarify the location of the high turbulent content regions in figure 13, figure 14 presents the wall-normal distributions of $\left\langle v^{\prime 2}\right\rangle,\left\langle u^{\prime} v^{\prime}\right\rangle$ and $\left\langle P_{12}\right\rangle$ for both halves of the corrugation. The cross sections are displayed in figure 1 . The results are also compared with those of the plane channel flow. A maximum point (absolute values) can be recognized in normal distributions of $\left\langle v^{\prime 2}\right\rangle$ and $\left\langle u^{\prime} v^{\prime}\right\rangle$. These peaks correspond to the shear layer created above the recirculation bubble (see figure $13(\mathrm{c})$ ). The high wall-normal gradient of the streamwise velocity, $\partial\langle u\rangle / \partial y$, increases the magnitude of the production term in this region, which leads to an increase of the magnitude of the Reynolds shear stress in this region. Around the separation point (14 (a) and (c)), the values of both $\left\langle v^{\prime 2}\right\rangle$ and $\left\langle u^{\prime} v^{\prime}\right\rangle$ are small and close to those in the plane channel, whereas in the second half and close to the reattachment point (14 (b) and (d)), the near wall values increase and become about five times higher than in the plane channel.

Considering figures 13 and 14, a small positive region can be recognized near the wall for both $\left\langle u^{\prime} v^{\prime}\right\rangle$ and $\left\langle P_{12}\right\rangle$, where for $\left\langle u^{\prime} v^{\prime}\right\rangle$ the thickness of the region is almost constant in the streamwise direction, whereas for $\left\langle P_{12}\right\rangle$ the thickness depends on the streamwise location. These positive regions are more clearly shown in figure 15. Regions of positive shear stresses have also been reported in Hudson et al. [1] for the fully wavy channel. They noted that the positive values of $\left\langle u^{\prime} v^{\prime}\right\rangle$ disappear if $\left\langle u^{\prime} v^{\prime}\right\rangle$ is calculated in a $s-n$ coordinate system aligned with the wall [1]. In the present study, the shear stresses were transformed to the $s-n$ coordinate system but no significant difference was observed. Since the positive values of $\left\langle u^{\prime} v^{\prime}\right\rangle$ and $\left\langle P_{12}\right\rangle$ appear in different locations (see figure 15 (a) and (b)), the positive values of $\left\langle u^{\prime} v^{\prime}\right\rangle$ must be produced by another source term. Figure 
16 presents the averaged wall-normal distributions of the convection $\left(\left\langle C_{12}\right\rangle\right)$, production $\left(\left\langle P_{12}\right\rangle\right)$, diffusion $\left(\left\langle D_{12}\right\rangle\right)$ and pressure-strain $\left(\left\langle\Pi_{12}\right\rangle\right)$ terms of the shear stress equation at the reattachment point $(x=1.1)$. The distributions are plotted for both the entire cross-section (figure 16 (a)) and close to the wall (16 (b)). According to these figures, a positive region is created close to the wall both for the pressure-strain and the convection terms, but the pressure-strain is much larger than the convection. Hence it can be concluded that the positive shear stress is created by the pressure-strain term. This is further confirmed in figure 17 which presents contours of the pressure-strain and the convection terms in the wavy part of the channel. As can be seen, a large positive region of the pressure strain term appears around the reattachment point, which corresponds to the region of positive $\left\langle u^{\prime} v^{\prime}\right\rangle$.

Figure 18 presents the streamwise distributions of maximum cross sectional values for the normal Reynolds stresses $\left(\left\langle u^{\prime 2}\right\rangle,\left\langle v^{\prime 2}\right\rangle\right.$ and $\left.\left\langle w^{\prime 2}\right\rangle\right)$ and, as can be seen, in all cases the maximum values appear around the reattachment point. Comparison of these maximum values with the corresponding values of the plane channel, displayed as three symbols on the right edge in figure 18, indicates that the predicted values of $\left\langle v^{\prime 2}\right\rangle$ and $\left\langle w^{\prime 2}\right\rangle$ in the smooth part of the wavy channel are close to those for the plane channel while, for $\left\langle u^{\prime 2}\right\rangle$, the values in the smooth part the wavy channel are smaller than those in the plane channel.

\subsubsection{Thermal field characterization}

Figures 19 and 20 present the normal distributions of the averaged temperature for different cross sections in the streamwise direction at $\operatorname{Pr}=0.71$ and 
$\operatorname{Pr}=3.5$, respectively. The results are compared with the $D N S$ results of the present study for the plane channel flow. Similar to what was described for the averaged velocity distributions, two distinct thermal regions appear in the temperature distributions: one high gradient region close to the wall, as the thermal inner region, and one almost constant gradient region, as the thermal outer region. At $\operatorname{Pr}=0.71$ (figure 19), the maximum thickness of the thermal inner region appears near the separation point $(x=0.4)$, which means that the maximum thermal boundary layer thickness (or the minimum Nusselt number, see figure 11) occurs near the separation point and that the thickness is also greater than that for the plane channel flow, see figure 19 (a). The thickness of the inner region starts to decrease when approaching the center of the corrugation, which indicates the thinning of the thermal boundary layer in this region and the minimum thickness (or maximum Nusselt number, see figure 11) occurs near the reattachment point, see figure 19 (b). By a comparison of the temperature profiles in both halves of the corrugation in figure 19, it is observed that the decreasing rate of the thermal boundary layer thickness in the first half of the corrugation (19 (a)) is higher than the second one (19 (b)). For instance, the thicknesses of the inner region at $x=0.6$ and $x=0.8$ in the first half of the corrugation are 0.05 and 0.025 , respectively, while at $x=1$ and $x=1.2$ in the second half, they are almost the same and about 0.02. The averaged Nusselt number distribution in figure 11 also reveals that the variation of the Nusselt number between $x=0.6$ and $x=0.8$ is higher than between $x=1$ and $x=1.2$. Comparison of the results with the plane channel shows that the thermal boundary layer near the reattachment point is about three times thin- 
ner than that for the plane channel. After the reattachment point, the thermal boundary layer thickness increases again and, at $x=1.8$, in the smooth part of the wavy channel, the temperature gradient is very close to that for the plane channel. At $\operatorname{Pr}=3.5$ (figure 20), the temperature distributions in the whole channel and the behavior in the inner region for the cross sections between the separation and reattachment points are the same as for $\operatorname{Pr}=0.71$. However, the thermal boundary layer thickness is thinner (or the Nusselt number larger) in all cross sections, see figures 20 and 11. It should also be mentioned here that the definition of an inner and outer thermal layer is only relevant for the cross sections before the separation and after the reattachment points and does not include the sections inside the recirculation zone.

A detailed study of the thermal boundary layer behavior in various parts of the wavy channel at $\operatorname{Pr}=0.71$ and $\operatorname{Pr}=3.5$ is presented in figures 21 (a) and (b). According to the available definitions for the thermal boundary layer over a flat plate, the location of the thermal boundary layer edge is defined by $\delta_{T}=\left(\langle T\rangle-\left\langle T_{w}\right\rangle\right) /\left(\left\langle T_{i n f}\right\rangle-\left\langle T_{w}\right\rangle\right)$, where $\left\langle T_{w}\right\rangle$ and $\left\langle T_{\text {inf }}\right\rangle$ represent the wall and farfield temperatures, respectively. According to this definition, $\delta_{T}=0$ and $\delta_{T}=1$ represent the locations of wall and boundary layer edge, respectively. The same definition is applied in the present study, but instead of $\left\langle T_{i n f}\right\rangle$, the bulk temperature, $\left\langle T_{b}\right\rangle$, is used. Figures 21 (a) and (b) present the contours of $\delta_{T}$ in the near wall region for the two Prandtl numbers $\operatorname{Pr}=0.71$ and $\operatorname{Pr}=3.5$, respectively. The results predicted in this part confirm that the maximum thickness of the thermal boundary layer appears around the separation point and the minimum thickness occurs close to the reattachment point. These points 
are attributed to the locations of the maximum and minimum of the Nusselt number, respectively (see figure 11). A comparison of the contours indicates that, for both Prandtl numbers, the minimum and maximum thicknesses of the thermal boundary layer appear at the same place near the separation and reattachment zones. Thus, an increase in the Prandtl number decreases the thermal boundary layer thickness in the whole channel. However, the effect of the Prandtl number around the reattachment point is more significant than its effect near the separation point.

Figures 22 (a) and (b) present contours of the wall normal turbulent heat transfer and the corresponding heat transfer production $\left(\left\langle P_{2 T}\right\rangle\right)$ at $\operatorname{Pr}=3.5$, respectively. The wall normal heat transfer production is calculated as:

$$
P_{2 T}=-\left\langle u_{2}^{\prime} u_{i}^{\prime}\right\rangle \frac{\partial\langle T\rangle}{\partial x_{i}}-\left\langle u_{i}^{\prime} T^{\prime}\right\rangle \frac{\partial\left\langle u_{2}\right\rangle}{\partial x_{i}}
$$

As can be seen in figure 22, a high turbulent heat transfer region is identified around the separation point, which is related to the detached shear layer at this point. For both the wall normal heat transfer and its production, the maximum values appear at the same locations and extend downstream nearly to the center of the corrugation. More detailed features of the temperature fluctuations can be found in figures 23 and 24 . Figure 23 compares the root mean square of temperature fluctuations $\left(\left\langle T_{r m s}\right\rangle\right)$ at four positions located at the separation, the center of the recirculating zone, the reattachment and the smooth part of the channel. It is observed that the temperature fluctuations are largest at the separation point.

The streamwise variations of $\left\langle T_{r m s}\right\rangle$ at the wall for $\operatorname{Pr}=0.71$ and $\operatorname{Pr}=3.5$ 
in the wavy part of the channel are compared in figure 24 (a). For both Prandtl numbers, the maximum fluctuations appear around the separation point and the Prandtl number has a strong impact on the maximum values of the fluctuations, especially close to the separation point. Comparison of the values with the corresponding $R M S$ value of the plane channel, displayed as a symbol on the right edge in figure 24 (a), indicates that employing the corrugations on the wall significantly increases the temperature fluctuations near the wall. It should be noted that, for a better comparison, all the values for $\operatorname{Pr}=0.71$ are multiplied by a factor of five. According to the probability density function $(P D F)$ plot (see figure $24(\mathrm{~b})$ ), around the separation point, $x=0.4$, the fluctuations are stronger and have a wider range than those around the reattachment point, $x=1.2$. The values obtained for skewness and flatness at $x=0.4, x=0.8$, $x=1.2$ and $x=2$ are $-0.3697,0.4425,-0.1897,-0.3951$ and $4.1038,2.4976$, $2.6973,2.6284$, respectively. The flatness at $x=0.4$ is about two times higher than that at the other three points, which means that the fluctuations at $x=0.4$ include some large values, whereas the fluctuations at $x=0.8, x=1.2$ and $x=2$ are nearly in the same ranges (see figure 24 (b)).

Comparing the locations of the minimum and maximum Nusselt number with the corresponding regions for the thermal turbulent parameters indicates that, in spite of having a high content thermal fluctuation region around the separation point, the minimum Nusselt number appears in this region. As can be seen in figure 11, the maximum Nusselt number appears around the reattachment point where the thermal boundary layer has its minimum thickness while the thermal fluctuations have their minimum in this region. Thus, it can 
be concluded that the magnitude of the thermal turbulent fluctuations at the wall are not correlated with the Nusselt number.

\section{Conclusion}

Numerical results of turbulent flow and heat transfer enhancement in a threedimensional periodic, fully developed wavy channel with the constant heat flux boundary condition and $R e_{b}=10000$ are presented. Large Eddy Simulation $(L E S)$ and Direct Numerical Simulation $(D N S)$ have been applied to solve the flow and energy equations. The predicted results are compared with the DNS results of the present study for the plane channel at $R e_{\tau}=395$. In the present study, the following conclusions are drawn:

1. The predicted results show that the maximum value of $\langle N u\rangle$ and $\left\langle C_{f}\right\rangle$ are located at nearly the same locations (downstream of the reattachment point). The minimum value of $\langle N u\rangle$ occur near the separation point.

2. Regions of large thermal turbulence fluctuations at the wall do not coincide with large heat transfer.

3. The local comparison of $\langle N u\rangle$ for Prandtl numbers 0.71 and $\operatorname{Pr}=3.5$ indicates that the presence of corrugation in the channel causes different effects near the separation and reattachment points. The reduced magnitudes for both Prandtl numbers around the separation point are almost the same and about $40 \%$. For the reattachment point, there is an increase of $21 \%$ and $36 \%$ for $\operatorname{Pr}=0.71$ and 3.5 , respectively. 
4. Comparison of the effect of the thermal boundary layer and thermal turbulent production on the local distribution of $\langle N u\rangle$ shows that the maximum value of the Nusselt number appears around the reattachment point, where the thermal boundary layer has its minimum thickness; the thermal turbulent production is very small at this streamwise location.

5. The resolved shear stress, $\left\langle u^{\prime} v^{\prime}\right\rangle$, is - as expected - negative in the major part of the domain. However, small regions of positive resolved shear stress are found. In the literature it is argued that also in these regions it stays negative when transformed to a $s-n$ coordinate system aligned with the corrugated wall. In the present work it is shown that also in this coordinate system the resolved shear stress is positive.

6. It is found that the positive resolved shear stress is created by the pressurestrain term in the transport equation for $\left\langle u^{\prime} v^{\prime}\right\rangle$.

\section{Acknowledgment}

This work was carried out at Chalmers during a one-year research visit of the first author.

\section{References}

[1] J. D. Hudson, L. Dykhno, T. J. Hanratty, Turbulence production in flow over a wavy wall, Int. J. Experiments in Fluids, vol. 20, pp. 257-265, 1996. 
[2] P. Cherukat, Y. Na, and T.J. Hanratty, Direct numerical simulation of a fully developed turbulent flow over a wavy wall, J. Theoretical and Computational Fluid Dynamics, vol. 11, pp. 109-134, 1998.

[3] H. S. Choi, K. Suzuki,Large eddy simulation of turbulent flow and heat transfer in a channel with one wavy wall, Int. J. Heat and Fluid Flow, vol. 26, pp.681-694, 2005.

[4] R. J. Calhoun and R. L. Street, Turbulent flow over a wavy surface: Neutral case, J. Geophysical Research, vol. 106, pp. 9277-9293, 2001.

[5] A. Z. Dellil, A. Azzi, B. A. Jubran, Turbulent flow and convective heat transfer in a wavy wall channel, Int. J. Heat and Mass Transfer, vol. 40, pp. 793-799, 2004.

[6] H. S. Yoon, O. A. El-Samni, A. T. Huynh, H. H. Chun, H. H. Kim, A. H. Pham, I. R. Park, Effect of wave amplitude on turbulent flow in a wavy channel by direct numerical simulation, Int. J. Ocean Engineering, vol. 36, pp. 697-707, 2009.

[7] H. Kawamura, H. Abe and K. Shingai, DNS of turbulence and heat transport in a channel flow with different Reynolds and Prandtl numbers and boundary conditions, 3rd Int. Symp. on Turbulence, Heat and Mass Transfer, Japan, 2000.

[8] F. Nicoud and F. Ducros, Subgrid scale stress modelling based on the square of the velocity gradient tensor, Int. J. Flow, Turbulence and Combustion, vol. 62, pp. 183-200, 1999. 
[9] L. Davidson and S. H. Peng, Hybrid LES-RANS: A one-equation $S G S$ model combined with a $k-\omega$ for predicting recirculating flows, Int. J. Numerical Methods in Fluids, vol. 43, pp. 1003-1018, 2003. 


\section{Tables}

\begin{tabular}{|c|c|c|}
\hline$H$ & height of channel & 1 \\
\hline$L$ & channel pitch & $3.68 H$ \\
\hline$H_{1}$ & corrugation height & $0.2 H$ \\
\hline$W$ & width of channel & $0.5 H$ \\
\hline
\end{tabular}

Table 1: Geometrical parameters of the computational domain

\begin{tabular}{|c|c|c|c|c|}
\hline Grid & Grid1 & Grid2 & Grid3 & Grid4 (two-pitch) \\
\hline Node numbers & $170 \times 130 \times 34$ & $338 \times 130 \times 34$ & $170 \times 198 \times 66$ & $338 \times 130 \times 34$ \\
\hline$\Delta x_{\max }^{+}$ & 11 & 5.5 & 11 & 11 \\
\hline$\Delta y_{\min }^{+}$ & 0.25 & 0.25 & 0.25 & 0.25 \\
\hline$\Delta z_{\max }^{+}$ & 7.5 & 7.5 & 3.75 & 7.5 \\
\hline$\left\langle C_{f}\right\rangle$ & $4.17 \times 10^{-3}$ & $4.23 \times 10^{-3}$ & $4.29 \times 10^{-3}$ & $4.38 \times 10^{-3}$ \\
\hline$\langle N u\rangle$ & 53.6 & 48.8 & 52.1 & 52.7 \\
\hline
\end{tabular}

Table 2: Comparison of different grid resolutions and the effect on the averaged results. $\Delta x_{\max }^{+}, \Delta y_{\max }^{+}$and $\Delta z_{\max }^{+}$are computed using $u_{\tau}$ at $x=0$.

\begin{tabular}{|c|c|c|c|c|c|}
\hline Case & Grid number & $\left\langle u_{b}\right\rangle$ & $R e_{b}$ & $\left\langle C_{f}\right\rangle$ & $\langle N u\rangle$ \\
\hline Present study & $66 \times 130 \times 66$ & 18.1 & 14200 & $6.1 \times 10^{-3}$ & 35.9 \\
\hline Kawamura et al.[7] & $258 \times 130 \times 258$ & 17.7 & 14000 & $6.39 \times 10^{-3}$ & 37.5 \\
\hline
\end{tabular}

Table 3: DNS results of mean flow variables for the present study and Kawamura et al. [7] at $R e_{\tau}=395$ 


\section{Figures}

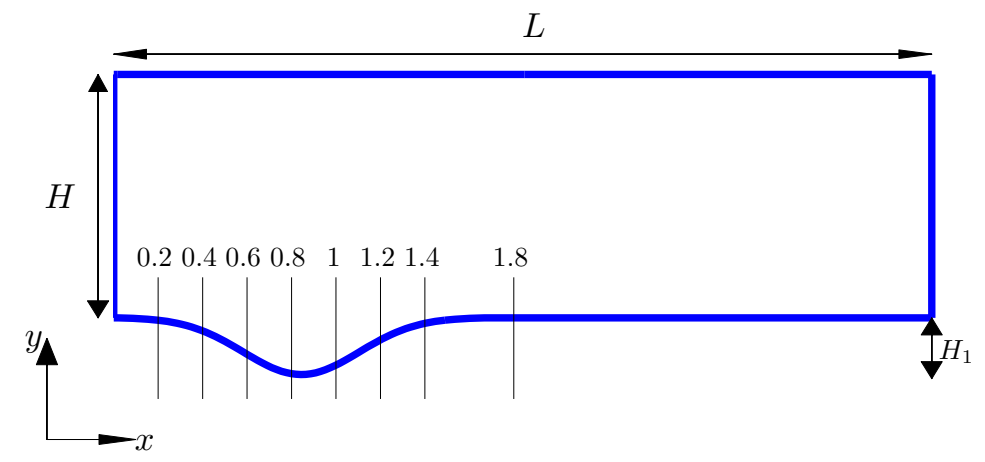

Figure 1: Schematic view of the computational domain indicating different cross sections in the streamwise direction

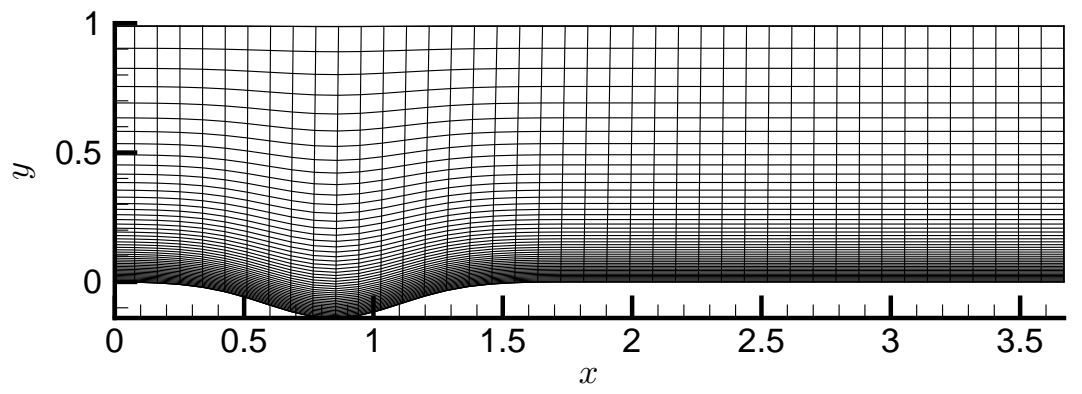

Figure 2: A schematic view of the grid resolution in the domain; every 4th gridline is shown 


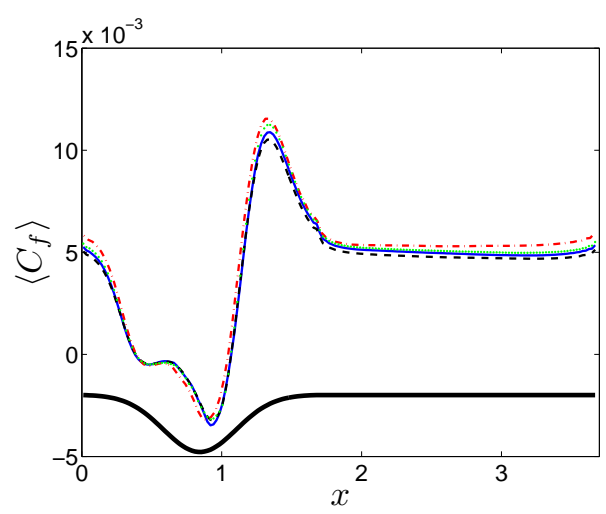

(a)

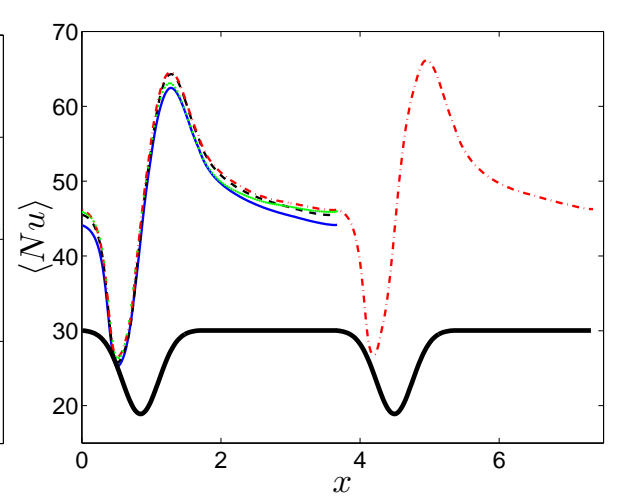

(b)

Figure 3: Comparison of the averaged (a) friction coefficient $\left(\left\langle C_{f}\right\rangle\right)$ and (b) Nusselt number $(\langle N u\rangle)$ distributions in the streamwise direction for different grids. The thick line in the lower part of the figures shows the contour of the bottom wall. _ _ - : grid $1 ; \ldots$ : $\operatorname{grid} 2 ; \ldots$ : grid $3 ; \ldots$. : grid4

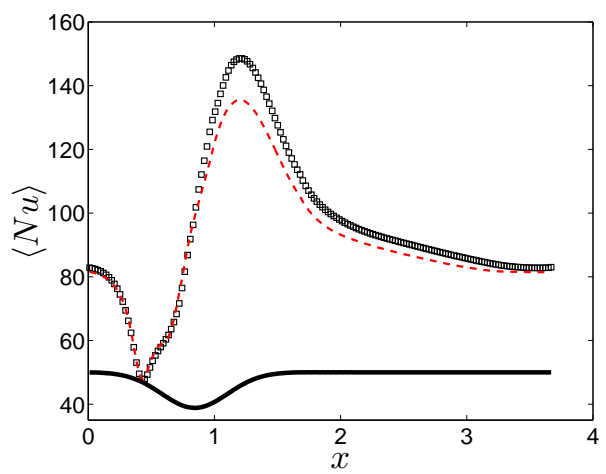

Figure 4: Comparison of $\langle N u\rangle$ for different grids at $\operatorname{Pr}=3.5$. The thick line in the lower part of the figure shows the contour of the bottom wall. - - - : grid3; $\square$ : grid5 


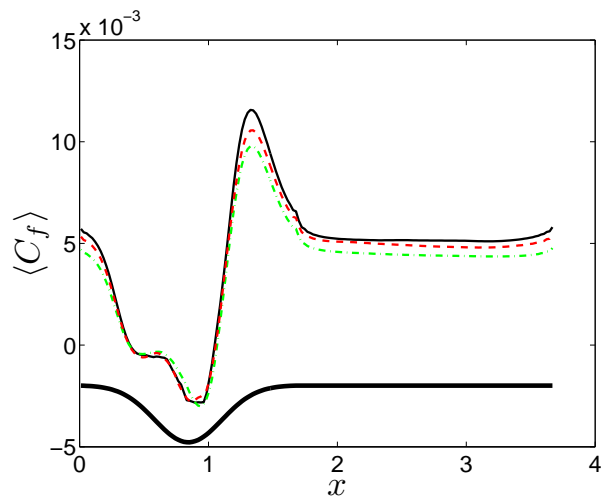

(a)

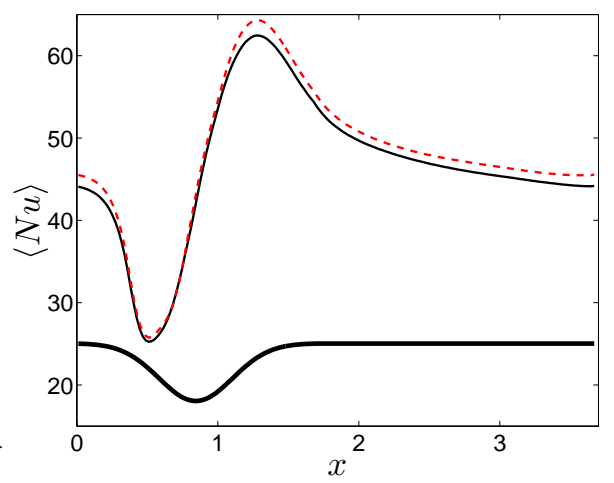

(b)

Figure 5: Comparison of the streamwise distributions of (a) $\left\langle C_{f}\right\rangle$ and (b) $\langle N u\rangle$ with the corresponding $D N S$ results. The thick line in the lower part of the figures shows the contour of the bottom wall. _ : DNS; - _: LES1; ... : $L E S 2$

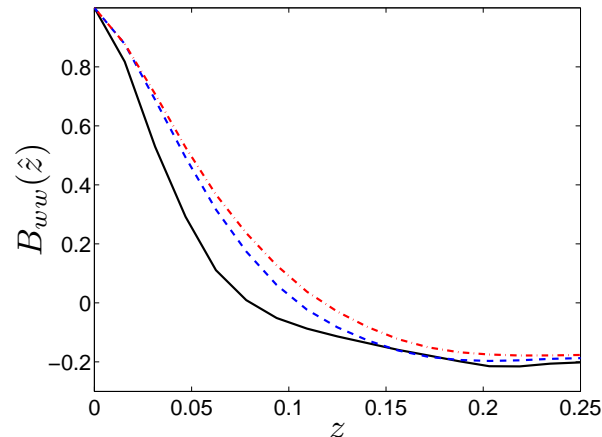

(a) $z_{\max }=0.5$

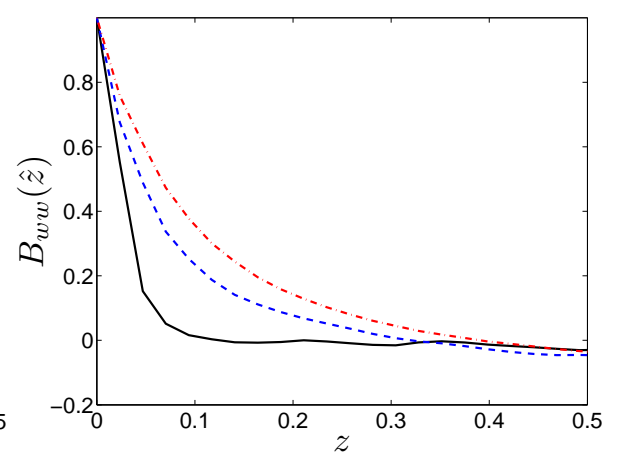

(b) $z_{\max }=1$

Figure 6: Comparison of two point correlation. $-: y=0.007 ;-\_-1: y=0.02$; $-.-: y=0.04$ 


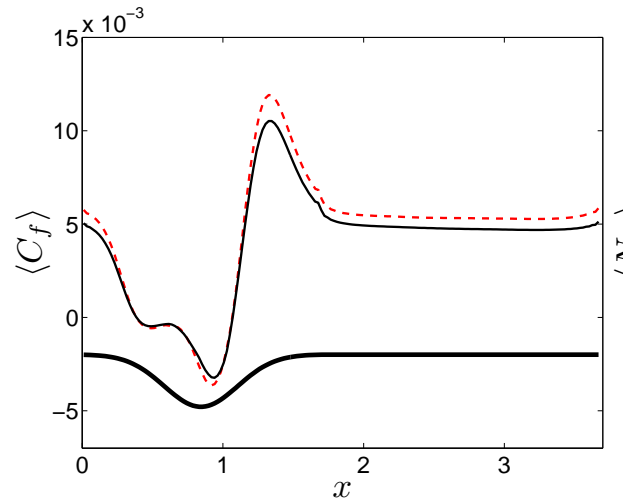

(a)

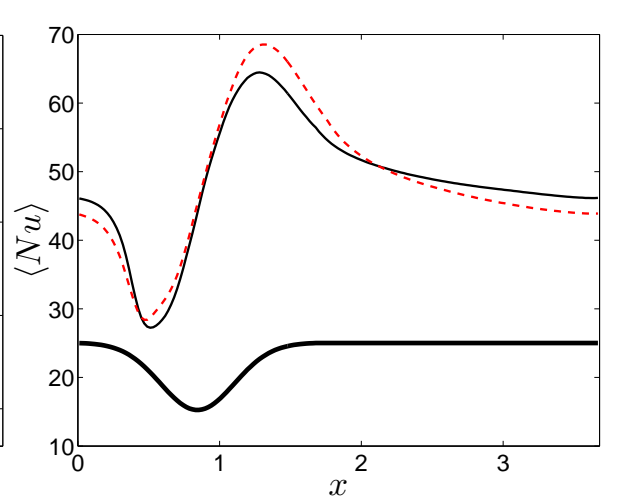

(b)

Figure 7: Comparison of the streamwise distributions of (a) $\left\langle C_{f}\right\rangle$ and (b) $\langle N u\rangle$ for two different channel width $z_{\max }=0.5$ and $z_{\max }=1$. The thick line in the lower part of the figures shows the contour of the bottom wall. - : $z_{\max }=0.5$; $-.-: z_{\max }=1$ 


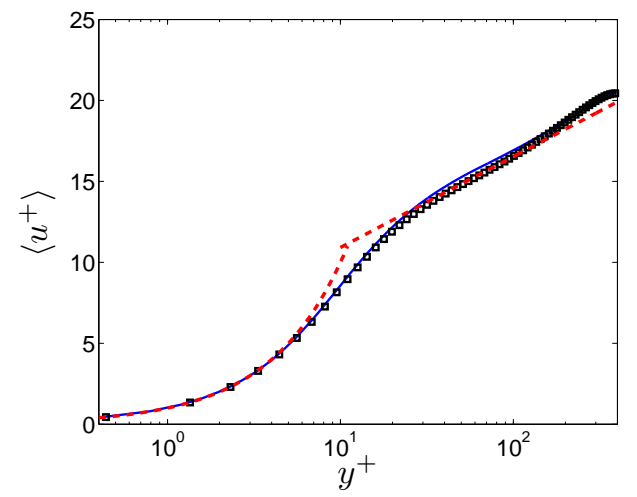

(a) Mean velocity distribution.

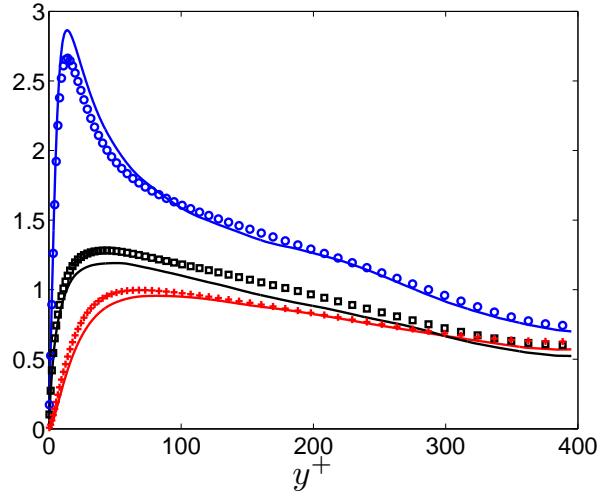

(b) Root mean square of velocity fluctuation. present study; $\square$ : DNS Kawamura et al. [7]; Smooth line: Present study; symbols: Kawamura et al. [7], ०: $\left\langle u^{\prime 2}\right\rangle ;+:\left\langle v^{\prime 2}\right\rangle ; \square:\left\langle w^{\prime 2}\right\rangle$

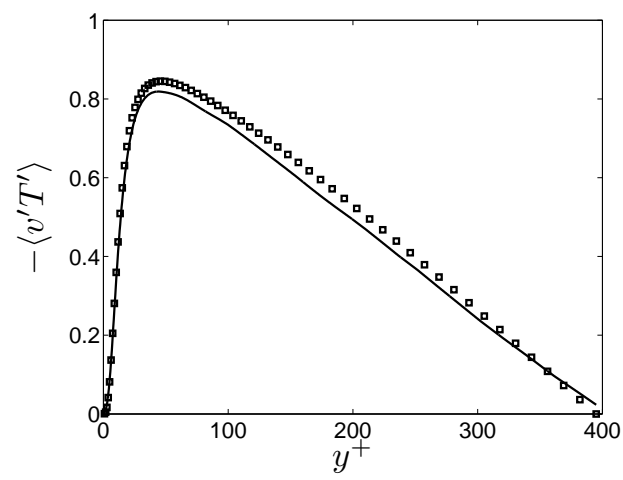

(c) The wall normal turbulent heat transfer.

— : Present study; $\square$ : Kawamura et al. [7]

Figure 8: Comparison of the $D N S$ results of the present study with the $D N S$ results of Kawamura et al. [7] at $R e_{\tau}=395$ 


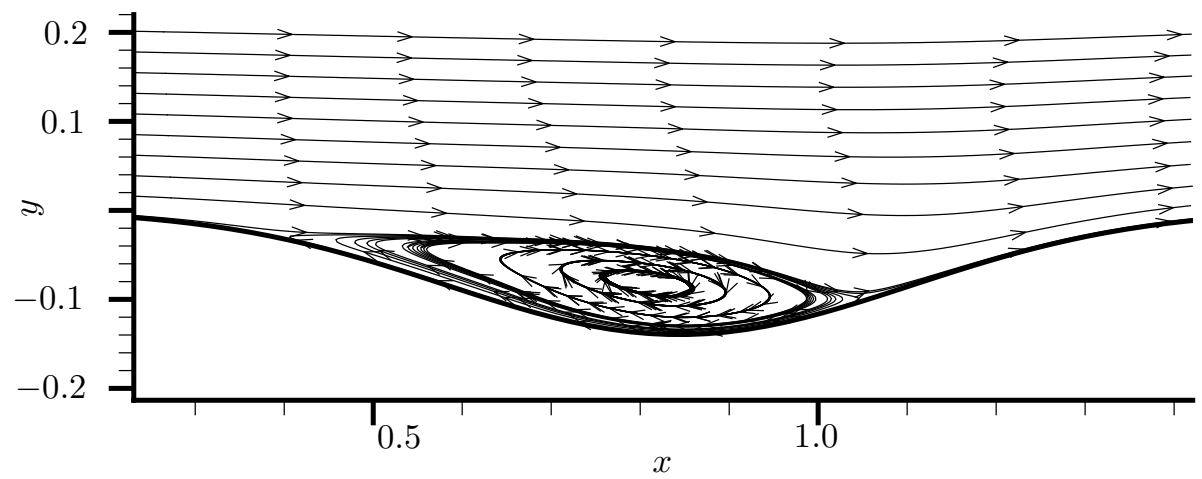

Figure 9: The averaged streamlines in the wavy (corrugated) part of the channel

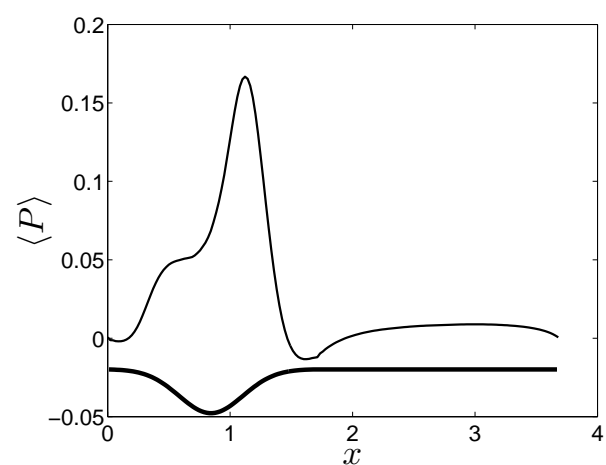

(a) Mean pressure in entire channel

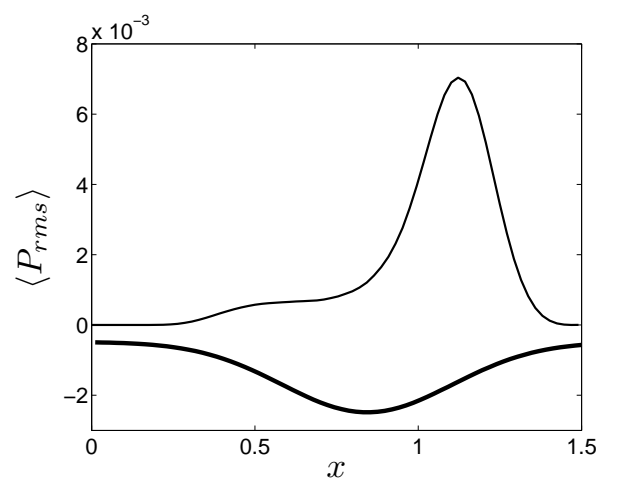

(b) $R M S$ pressure fluctuations in wavy part of the channel

Figure 10: The streamwise distributions of the averaged pressure $(\langle P\rangle)$ and pressure fluctuations $\left(\left\langle P_{r m s}\right\rangle\right)$ at the wall. The thick line in the lower part of the figures shows the contour of the bottom wall. 


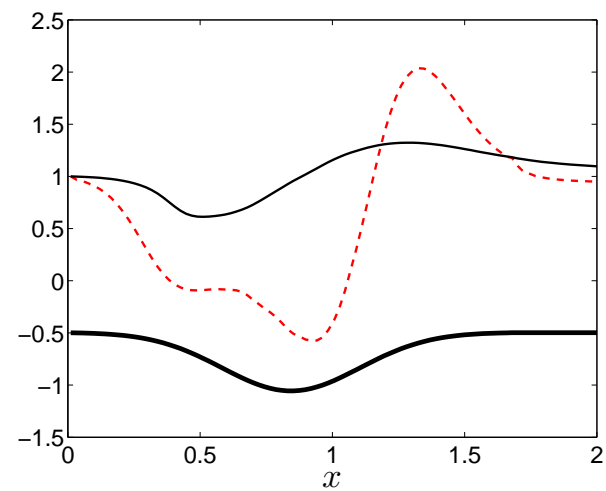

(a) Comparison of $\left\langle C_{f}\right\rangle /\left\langle C_{f_{x=0}}\right\rangle$ and $\langle N u\rangle /\left\langle N u_{x=0}\right\rangle$ at $\operatorname{Pr}=0.71$. The thick line in the lower part of the figure shows the contour of the bottom wall. $-\langle N u\rangle ;---$ : $\left\langle C_{f}\right\rangle$

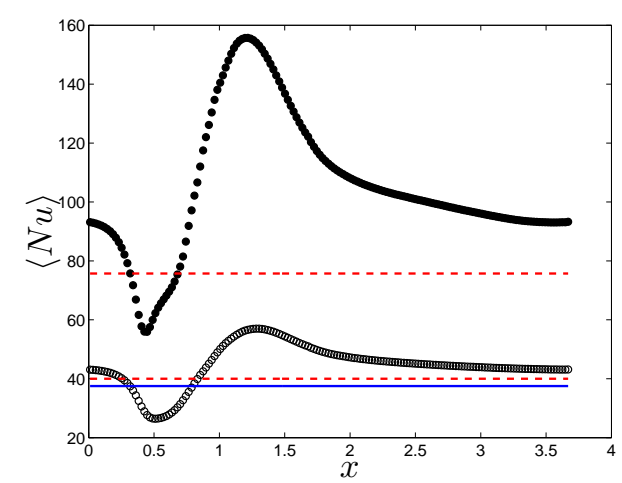

(b) Comparison of $\langle N u\rangle$ at $\operatorname{Pr}=0.71$ and 3.5. : DNS for plane channel, $\operatorname{Pr}=0.71 ;---$ : Analytical formulas for plane channel, $\operatorname{Pr}=0.71$ and 3.5 ; ○: LES for wavy channel, $\operatorname{Pr}=0.71$; $\bullet$ LES for wavy channel, $\operatorname{Pr}=3.5$

Figure 11: The streamwise distributions of the averaged Nusselt number and friction coefficient 


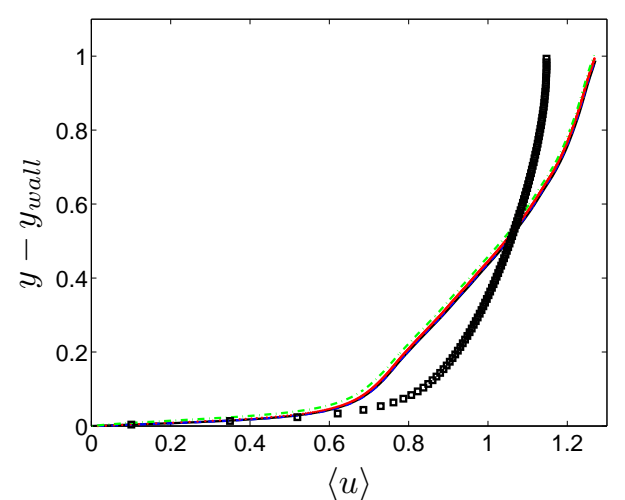

(a) Before the separation point. $-1: x=0$;

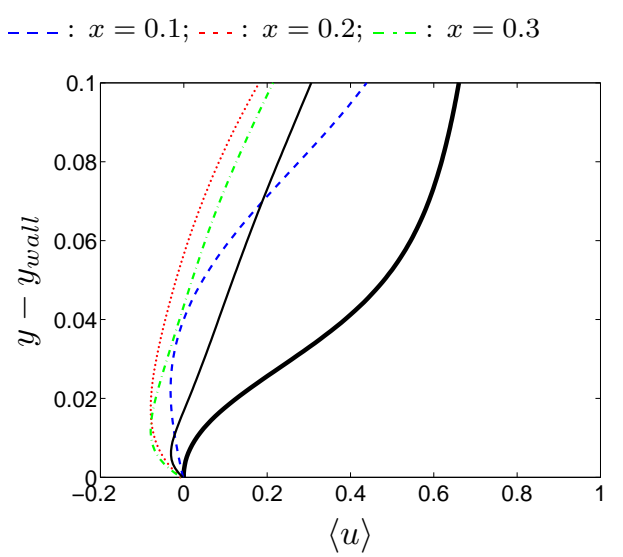

(c) In the recirculation zone.

$\longrightarrow x=0.4$

$-\_: x=0.6 ; \ldots: x=0.8 ;-\cdots: x=0.9$;

$-x=1$

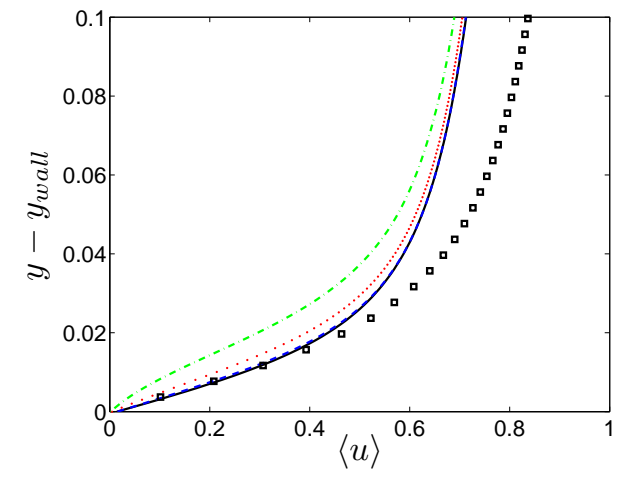

(b) Before the separation point, zoom near the wall. For legend, see Fig. 12(a)

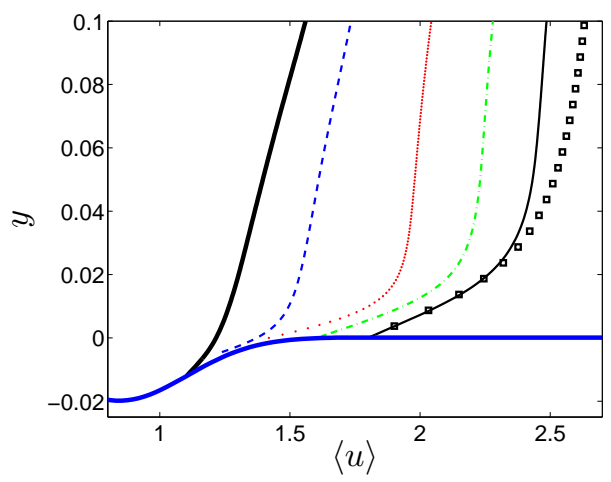

(d) After the reattachment point. $-x=1.1$;

$---: x=1.2 ;-.: x=1.4 ;-\cdots: x=1.6 ;-$ $x=1.8$; The thick blue line in the lower part of the figure shows the contour of the bottom wall

Figure 12: Averaged wall-normal velocity distribution $(\langle u\rangle)$ for different cross sections in the streamwise direction. $\square:\left\langle u / u_{b}\right\rangle_{D N S}$ of the present DNS of the plane channel. 


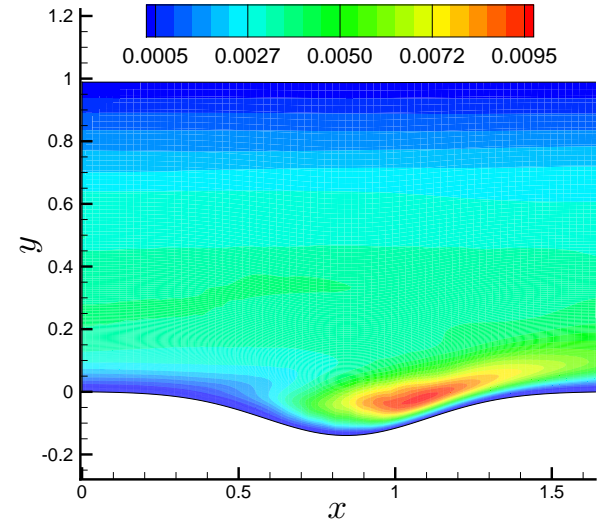

(a) $\left\langle v^{\prime 2}\right\rangle$

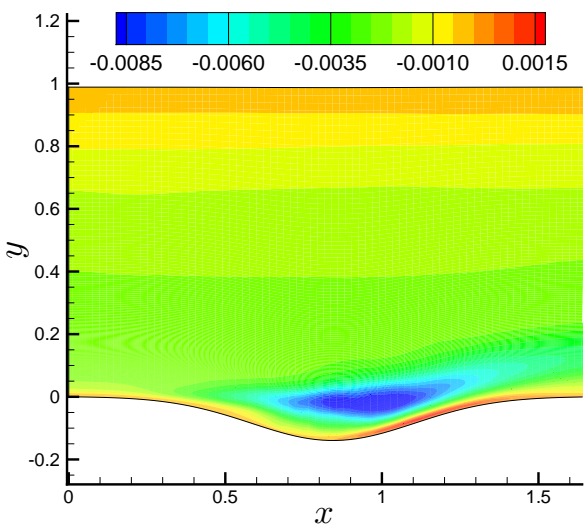

(b) $\left\langle u^{\prime} v^{\prime}\right\rangle$

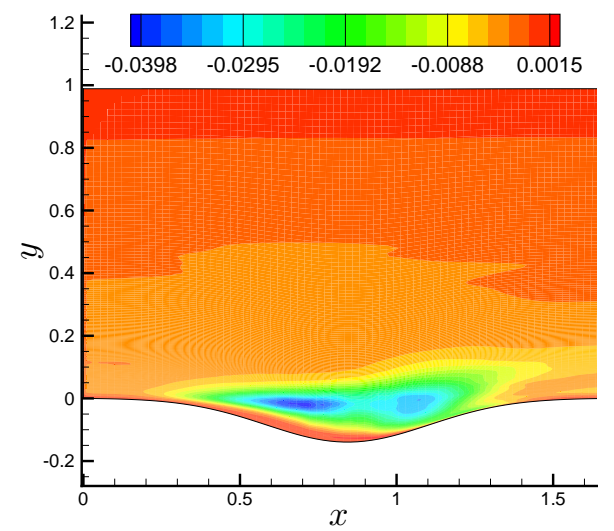

(c) $\left\langle P_{12}\right\rangle$

Figure 13: Contours of the averaged normal Reynolds stress $\left(\left\langle v^{2}\right\rangle\right)$, shear stress $\left(\left\langle u^{\prime} v^{\prime}\right\rangle\right)$ and shear stress production $\left(\left\langle P_{12}\right\rangle\right)$ in the wavy part of the channel 

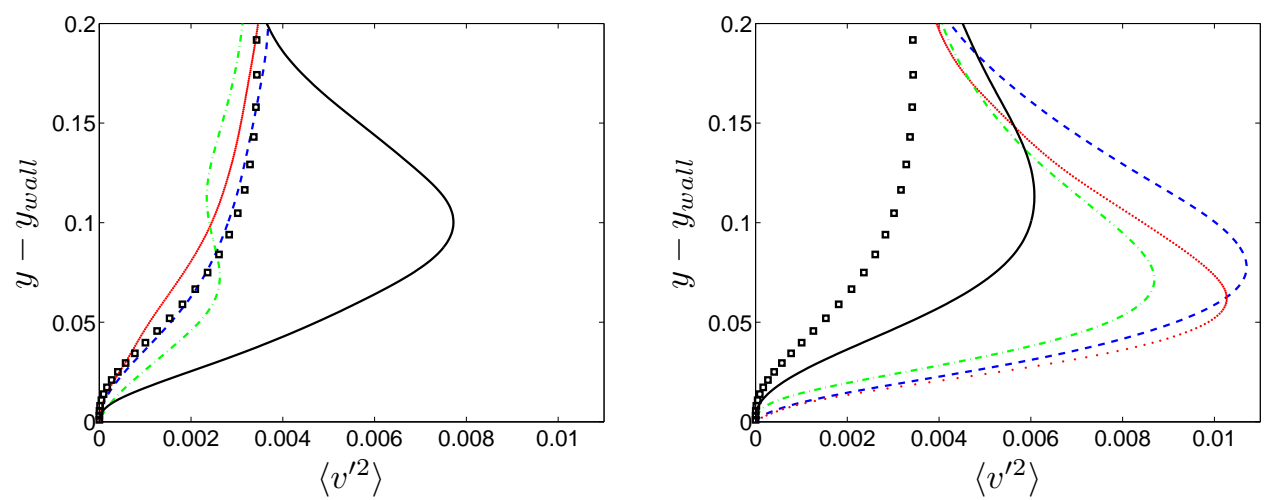

(a) First half of corrugation. - - - : $x=0.2 ; \ldots$ :

(b) Second half of corrugation. - - - : $x=1$;

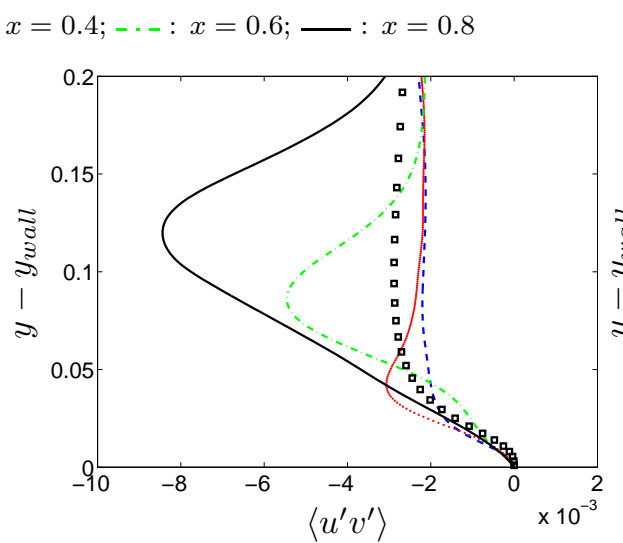

(c) First half of corrugation

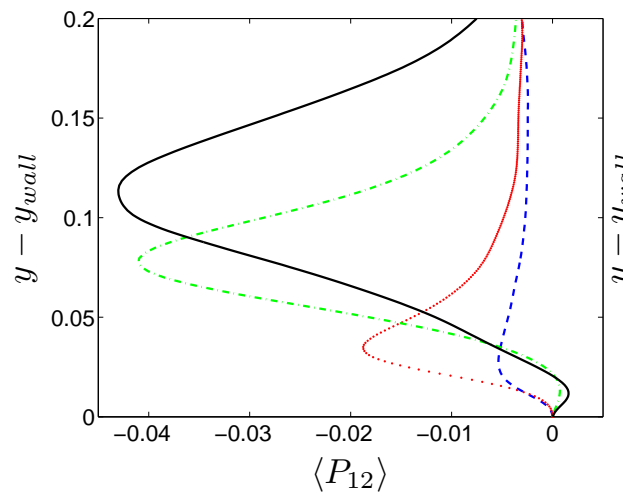

(e) First half of corrugation

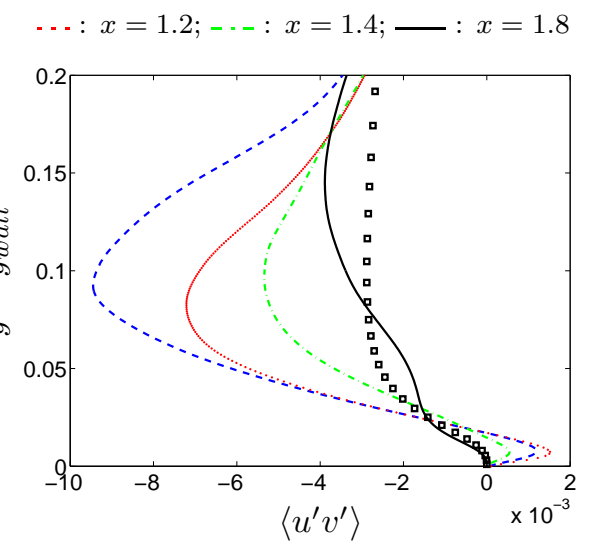

(d) Second half of corrugation

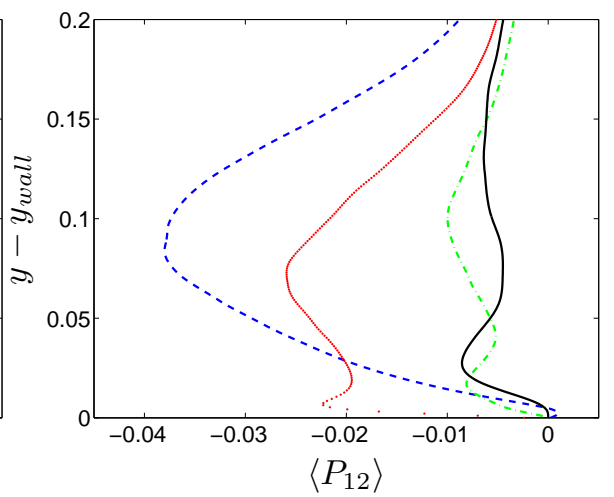

(f) Second half of corrugation

Figure 14: Comparison of the wall-normal distributions of normal Reynolds stress $\left(\left\langle v^{\prime 2}\right\rangle\right)$, shear stress $\left(\left\langle u^{\prime} v^{\prime}\right\rangle\right)$ and shear stress production $\left(\left\langle P_{12}\right\rangle\right)$ for the first and second halves of the corrugation. $\square$ : DNS results of the present study for plane channel (scaled with $u_{b}^{2}$ or $u_{b}^{3}$ ) 


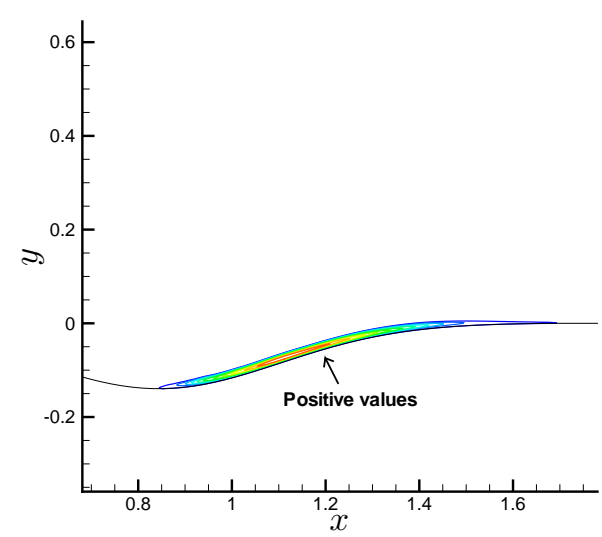

(a) $\left\langle u^{\prime} v^{\prime}\right\rangle$

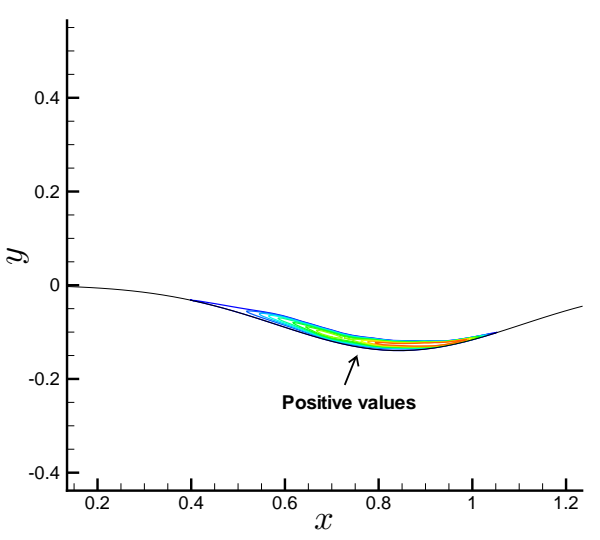

(b) $\left\langle P_{12}\right\rangle$

Figure 15: Positive-value regions of the averaged shear stress $\left(\left\langle u^{\prime} v^{\prime}\right\rangle\right)$ and shear stress production $\left(\left\langle P_{12}\right\rangle\right)$

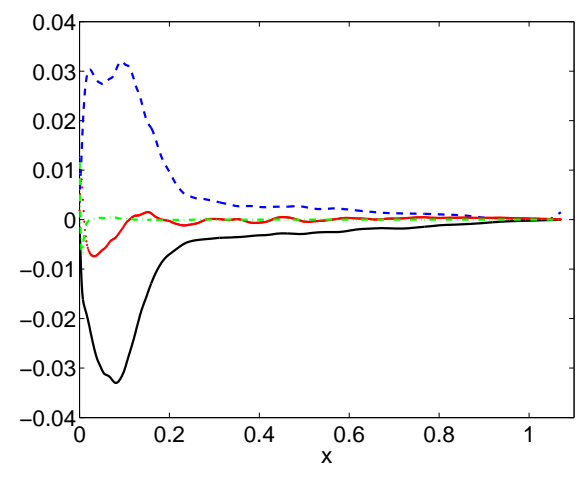

(a) In the entire channel

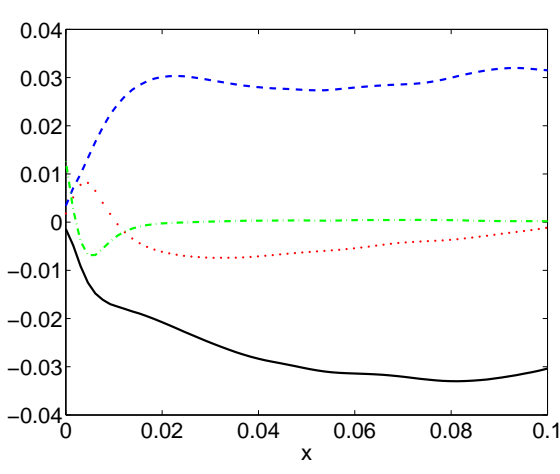

(b) Zoom near the wall

Figure 16: Various terms of shear stress equation at $x=1.1, \ldots$ : production $\left(\left\langle P_{12}\right\rangle\right) ;-\ldots$ - pressure-strain $\left(\left\langle\Pi_{12}\right\rangle\right) ; \ldots$ : convection $\left(\left\langle C_{12}\right\rangle\right) ;-.$. : diffusion $\left(\left\langle D_{12}\right\rangle\right)$ 


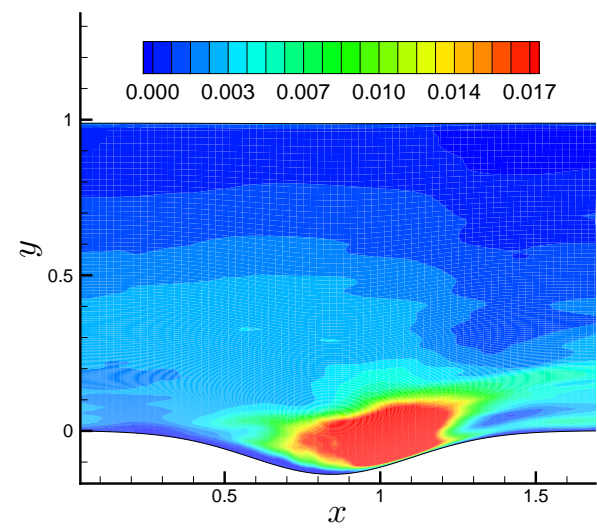

(a) $\left\langle\Pi_{12}\right\rangle$

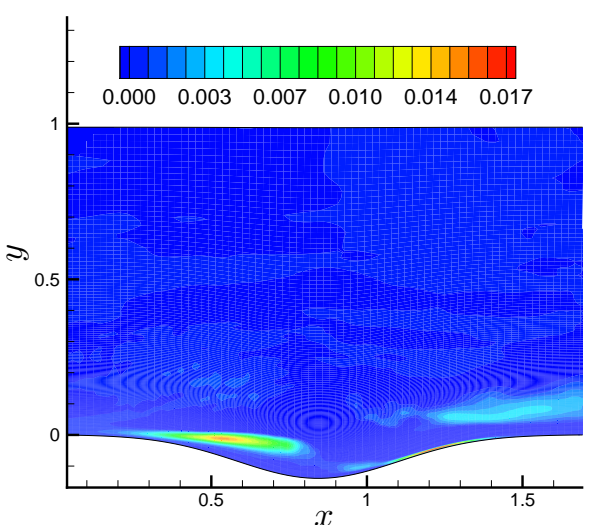

(b) $\left\langle C_{12}\right\rangle$

Figure 17: Contours of the averaged pressure strain $\left(\left\langle\Pi_{12}\right\rangle\right)$ and convection $\left(\left\langle C_{12}\right\rangle\right)$ in the wavy part of the channel

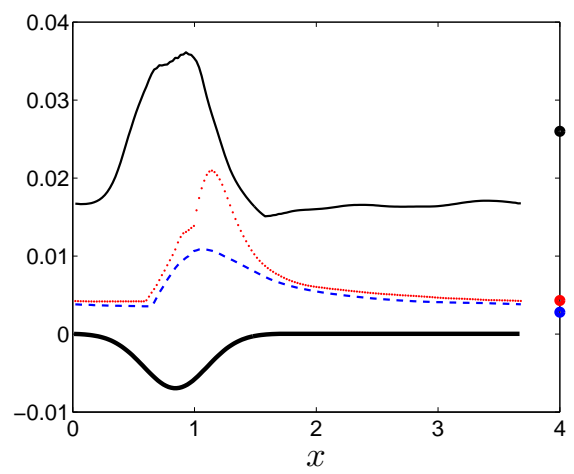

Figure 18: The streamwise distributions of the maximum cross sectional values for the Reynolds stresses. The thick line in the lower part of the figure shows the contour of the bottom wall. _- : $\left\langle u^{\prime 2}\right\rangle ;---:\left\langle v^{\prime 2}\right\rangle ; \ldots:\left\langle w^{\prime 2}\right\rangle$. The markers (at the far right) show the corresponding values for DNS of plane channel. 


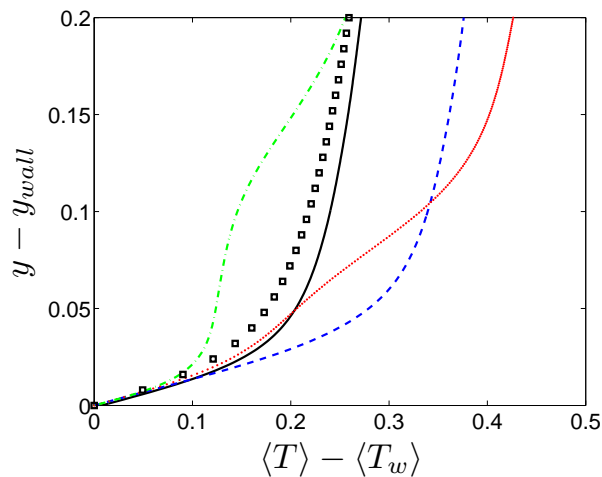

(a) First half of the corrugation. $-\frac{\square}{-}:=0.2$; $-\ldots$ - : $x=0.4 ; \ldots: x=0.6 ;-. .: x=0.8$

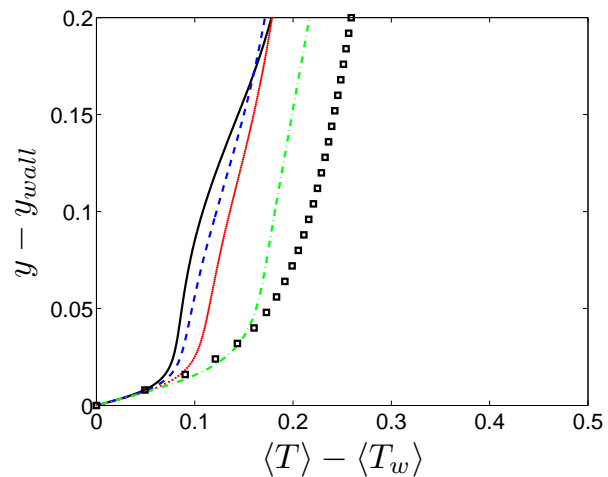

(b) Second half of the corrugation. $\_: x=1$; $-\_-: x=1.2 ;-.: x=1.4 ; \ldots-.: x=1.8$

Figure 19: Averaged wall-normal temperature distribution $\left(\langle T\rangle-\left\langle T_{w}\right\rangle\right)$ in the streamwise direction at $\operatorname{Pr}=0.71 . \square: D N S$ results of the present study for the plane channel (scaled with $u_{b}$ )

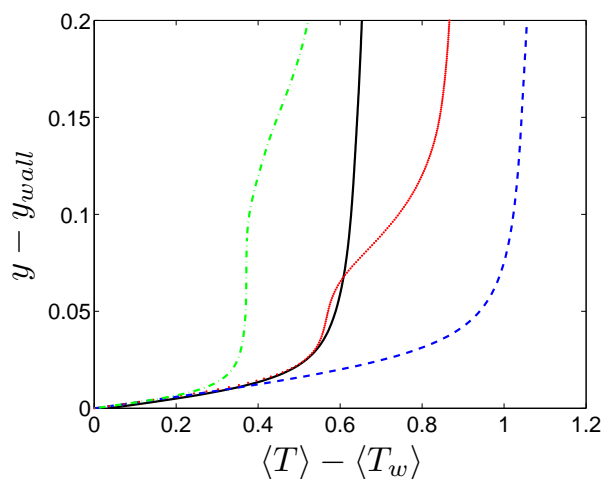

(a) First half of the corrugation

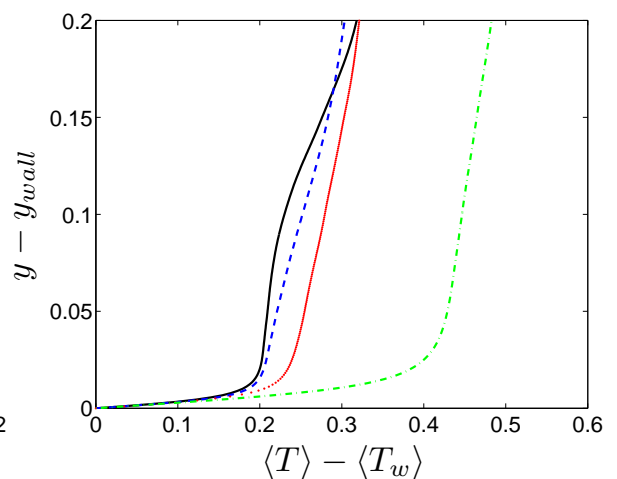

(b) Second half of the corrugation

Figure 20: Averaged wall-normal temperature distribution $\left(\langle T\rangle-\left\langle T_{w}\right\rangle\right)$ in the streamwise direction at $\operatorname{Pr}=3.5$; figure labels are presented in figure 19 


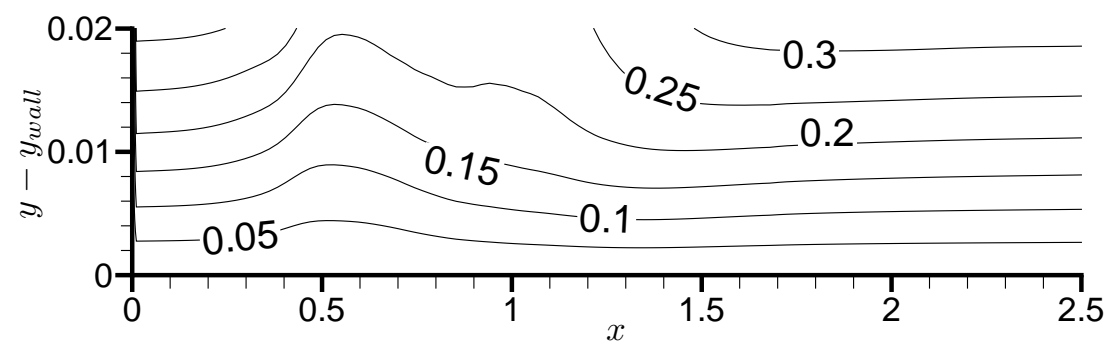

(a) $\operatorname{Pr}=0.7$

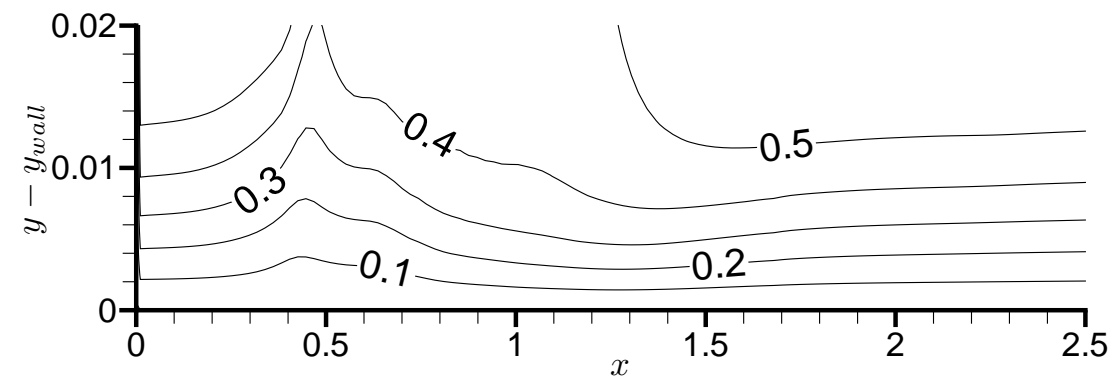

(b) $\operatorname{Pr}=3.5$

Figure 21: Contours of $\delta_{T}$ in the wavy part of channel for $\operatorname{Pr}=0.7$ and $\operatorname{Pr}=3.5$ 


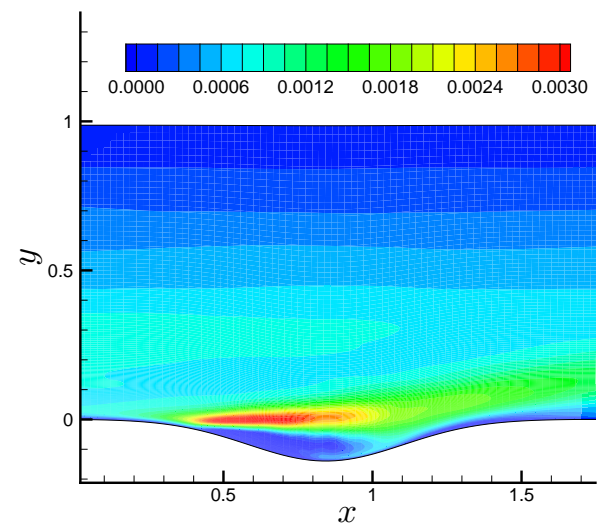

(a) $\left\langle v^{\prime} T^{\prime}\right\rangle$

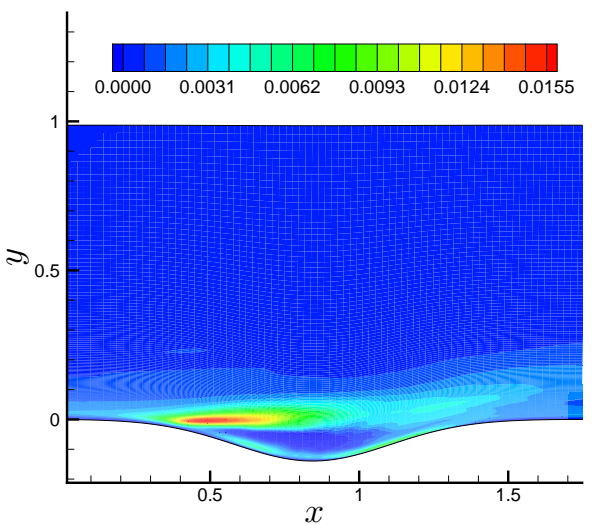

(b) $\left\langle P_{2 T}\right\rangle$

Figure 22: Contours of turbulent wall-normal heat transfer $\left(\left\langle v^{\prime} T^{\prime}\right\rangle\right)$ and heat transfer production $\left(\left\langle P_{2 T}\right\rangle\right)$ at $\operatorname{Pr}=3.5$

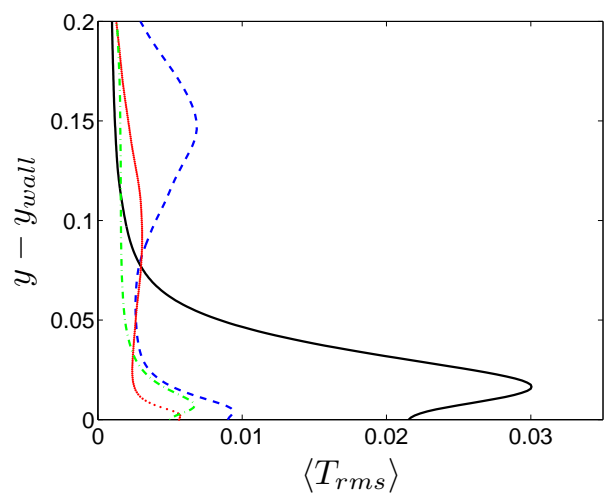

Figure 23: Wall-normal distribution of temperature fluctuations $\left(\left\langle T_{r m s}\right\rangle\right)$ at $\operatorname{Pr}=3.5 .-1 \mathrm{~L}=0.4 ;---: x=0.8 ; \ldots: x=1.2 ;-. .: x=2$ 

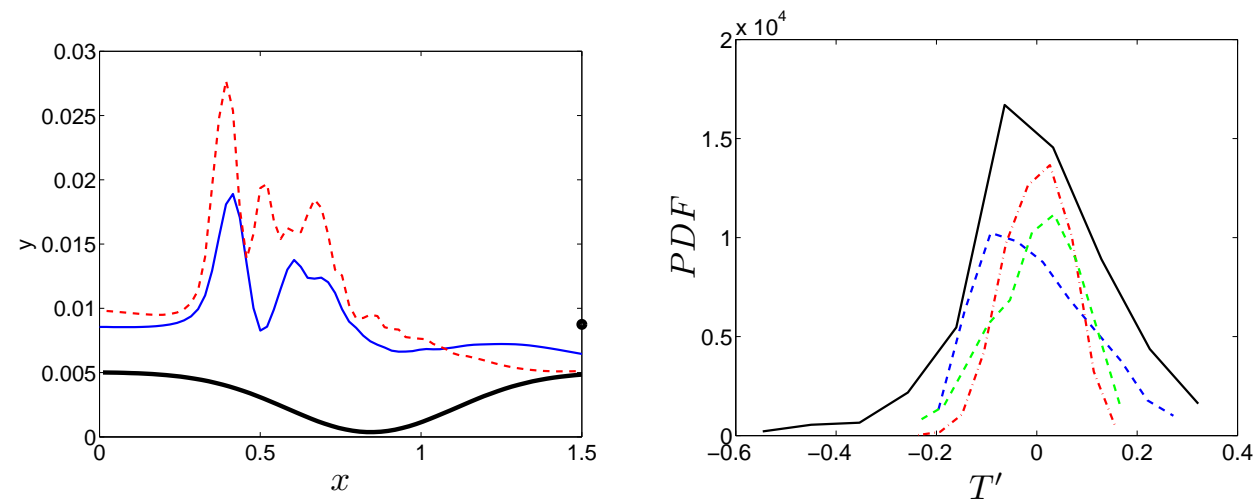

(a) — : $\operatorname{Pr}=0.71$ (multiplied by 5$) ;---$ :

(b) - : $x=0.4 ;---:$ : $x=0.8 ; \ldots: x=1.2$;

$\operatorname{Pr}=3.5 ; \bullet$ : fully developed channel flow ob-

$-\cdot: x=2$

tained by DNS (multiplied by 5 ). The thick line

in the lower part of the figure shows the contour

of the bottom wall.

Figure 24: (a) Comparison of the streamwise variation of the wall temperature fluctuations $\left(\left\langle T_{r m s}\right\rangle\right)$ in the wavy part of the channel at $\operatorname{Pr}=0.71$ and 3.5. (b) Probability density function $(P D F)$ at $\operatorname{Pr}=3.5$ 